\title{
Structure Formation and Non Linear Electrodynamics with Attendant Changes in Gravitational Potential and Its Relationship to the 3 Body Problem
}

\author{
Andrew Walcott Beckwith \\ College of Physics, Chongqing University, Huxi Campus, Chongqing, China \\ Email: Rwill9955b@gmail.com
}

How to cite this paper: Beckwith, A.W. (2018) Structure Formation and Non Linear Electrodynamics with Attendant Changes in Gravitational Potential and Its Relationship to the 3 Body Problem. Journal of High Energy Physics, Gravitation and Cosmology, 4, 779-786.

https://doi.org/10.4236/jhepgc.2018.44043

Received: June 19, 2018

Accepted: October 21, 2018

Published: October 24, 2018

Copyright $\odot 2018$ by author and Scientific Research Publishing Inc. This work is licensed under the Creative Commons Attribution International License (CC BY 4.0).

http://creativecommons.org/licenses/by/4.0/

\section{(c) (i) Open Access}

\begin{abstract}
We find that having the scale factor close to zero due to a given magnetic field value, an early universe magnetic field affects how we would interpret Mukhanov's chapter on "self reproduction of the universe". We extend such arguments, and refer to the possibility of modified gravity. We hope that some of the issues raised by Kobayashi and Seto as to allowed inflation models may be addressed, once further refinement of these preliminary results commences. We close with statements as to the value of $\alpha$ in a gravitational potential proportional to $r^{-\alpha}$ and how this adjustment affects the 3 body problem.
\end{abstract}

\section{Keywords}

Inflation, Modified Gravity, 3 Body Problem

\section{Introduction}

Part 1: We first of all will look at how a scale factor is affected by the NLED paradigm which in fact also is linked to the idea of "self reproduction" as given in [1], which is a different way as to outline how this affects the evolution of density in the early universe.

In doing so, we recount the work done by the author in [2] and summarize it in the section below.

Part 2: Next, after having done this, we give a description of an equation for setting the value of $\alpha$ in a gravitational potential proportional to $r^{-\alpha}$. This $\alpha$ has real and complex values, unlike the Newtonian real value.

Part 3: We then summarize what this has to do with possible revisions of the 
three-body-problem [3] with a particular mention as to how this would affect material as of page 141 and beyond in [3] with implications as to astrophysics.

Having said that we will introduce material which is congruent to references [1], [2], [3], and [4] for review, we will begin with a recapitulation of the main points of [2] given below. In doing so, it should be understood that the non linear cosmological electrodynamics, is a means toward providing more material which will allow for self reproduction of cosmological structure as given in [1].

\section{Part 1, We First of All Will Look at How a Scale Factor Is Affected by the NLED' in a Re Capitulation of Reference [2]}

What we are asserting is. in [1] as also stated in [2] there exists a scaled parameter $\lambda$, and a parameter $a_{0}$ which is paired with $\alpha_{0}$. For the sake of argument, we will set the $a_{0} \propto \sqrt{t_{\text {Planck }}}$, with $t_{\text {Planck }} \sim 10^{-44}$ seconds. Also, $\Lambda$ is a cosmological "constant" and is in [2], [5] via: use of what is given in [2] we have the following abbreviations, namely

$$
\alpha_{0}=\sqrt{\frac{4 \pi G}{3 \mu_{0} c}} B_{0}, \text { and } \lambda=\Lambda c^{2} / 3
$$

Then if $t_{\min } \approx t_{0} \equiv t_{\text {Planck }} \sim 10^{-44} \mathrm{~s}$ as our time scale, we can write that we have a minimum scale factor by [1] and [2] we have that

$$
a_{\min }=a_{0} \cdot\left[\frac{\alpha_{0}}{2 \lambda}\left(\sqrt{\alpha_{0}^{2}+32 \lambda \mu_{0} \omega B_{0}^{2}}-\alpha_{0}\right)\right]^{1 / 4}
$$

We should look at the background given in references [5]-[10] as all these references in part are influencing our structure formation ideas. Note also that Equation (4) is of the order of about $10^{-55}$, i.e. 33 orders of magnitude smaller than the square root of Planck time, which is useful in justifying the relative size of the $\Lambda$ which is done in [2] Non withstanding the temperature variation in reference [10] for the cosmological Hubble parameter, we also can reference what is done in references [2], and [5] namely a specific temperature variation which is in the spirit of [10] which we write as

$$
\Lambda(t) \sim\left(H_{\text {inflation }}\right)^{2} \text { which implies } \Lambda_{\max } \sim c_{2} \cdot T_{\text {temperature }}^{\tilde{\beta}}
$$

Our assumption though is that initially, at the beginning of creation of structure is that what we call Equation (3) is approximately constant. Therefore in lieu of [2] we thereby obtain the initial density behavior. which is commensurate with setting

$$
\left(\rho \sim a^{-4}\right)^{-1} \sim a^{4} \sim \frac{16}{3} \cdot c_{1}^{-1} \cdot B^{-4} \sim a_{0}^{-4} /\left[\frac{\alpha_{0}}{2 \lambda}\left(\sqrt{\alpha_{0}^{2}+32 \lambda \mu_{0} \omega B_{0}^{2}}-\alpha_{0}\right)\right]
$$

Note that from [2] we have material which is in common with [11] [12] [13] and then we look at

$$
\frac{\Delta H}{H} \sim \Omega_{m}^{2} h^{2} \Delta_{\Re} \sim 10^{-5}
$$


This is a re duplication of information, as given by Lloyd, in [14] which we cited in [2] for which we have

$$
\begin{aligned}
& \text { \# operations } \sim \rho_{\text {crit }} \times t^{4} \sim\left(t / t_{p}\right)^{2} \\
& \Leftrightarrow \rho_{\text {crit }} \sim 1 / t^{2} \propto \rho_{\gamma}=\frac{16}{3} \cdot c_{1} \cdot B^{4} \\
& \Leftrightarrow \text { \# operations } \sim\left(t / t_{p}\right)^{2} / B^{8} \\
& \sim 1 /\left(t_{p} B^{4}\right) \sim 1 / B^{4}
\end{aligned}
$$

Note that this also has similarities to [12]. If we have such a treatment of information as given by Lloyd [14], then, in terms of what may be generated and show up in the CMBR we may see using [12] [13] [14]

$$
\Delta H(\text { thermal }) \sim H_{\text {base-line }} \cdot(\# \text { operations })^{1 / 4} \cdot 10^{-5} \cdot \sqrt{t / t_{\text {Planck }}}
$$

We then have ,perhaps, the beginning of how NLED may impact fluctuations in $\mathrm{H}$, which in turn may lead to the issue we started our discussion over. Equation (7) We shall next investigate how part 1 and its results affect gravitational potential behaviour, in the Pre Planckian to Planckian universe evolution, next.

\section{Part 2, the Problem of the $\alpha$ in a Gravitational Potential Proportional to $r^{-\alpha}$}

In order to review this, we need to look at reference [15] where we can use the following treatment of the Klein Gordon equation which we write as

$$
\begin{aligned}
& \ddot{\varphi}_{k}+3 H \dot{\varphi}_{k}+\frac{k^{2}}{a^{2}} \varphi_{k}=0 \\
& \& \varphi_{k} \approx \frac{H \tau}{\sqrt{2 k}} \cdot\left(1+(i k \tau)^{-1}\right) \cdot \exp [-i k \tau] \\
& \& \tau=-H^{-1} \cdot \exp [-H \cdot t]
\end{aligned}
$$

Here, $\mathrm{k}$ is the value of wave number, and $\mathrm{H}$ is assumed, in the early universe to be a constant. The net result is that $k=2 \pi / \lambda$, with $\lambda$ proportional to the "width" of a would be pre universe "bubble" as seen in [16] place of a singularity, and also that one would have, for a constant H, during this time as seen by [17], [18]

$$
\begin{aligned}
& H=\sqrt{\frac{8 \pi G}{3} \cdot \rho-\frac{\kappa}{a^{2}}} \\
& \rho=\text { 'energy-density' } \\
& \kappa=\text { 'curvature' }
\end{aligned}
$$

Further use of [18] will lead to the situation that

$$
\begin{aligned}
& H=\sqrt{\frac{8 \pi G}{3} \cdot \rho-\frac{\kappa}{a^{2}}}=\sqrt{\frac{8 \pi G}{3}} \cdot \sqrt{\rho-\frac{3}{8 \pi G} \cdot \frac{\kappa}{a^{2}}} \\
& \approx \sqrt{\frac{8 \pi G}{3}} \cdot \sqrt{V(\varphi)-\frac{\dot{\varphi}^{2}}{2}} \\
& \Leftrightarrow \frac{\dot{\varphi}^{2}}{2}=\left(\frac{3}{8 \pi G} \cdot \frac{\kappa}{a^{2}}+V(\varphi)\right)-\rho
\end{aligned}
$$


Chaotic inflation would be using the approximation that

$$
V(\varphi) \approx \frac{k^{2}}{a^{2}} \cdot \varphi^{2}
$$

Use the approximation that the time derivative is $\mathrm{d} / \mathrm{d} \tau$, and $\varphi \equiv \varphi_{k}$, and if so, then

$$
\begin{aligned}
& \frac{\dot{\varphi}_{k}^{2}}{2}=\left(\frac{3}{8 \pi G} \cdot \frac{\kappa}{a^{2}}+\frac{k^{2}}{a^{2}} \cdot \varphi_{k}^{2}-\rho\right) \\
& \& \frac{\dot{\varphi}_{k}^{2}}{2}=\left(\frac{3}{8 \pi G} \cdot \frac{\kappa}{a^{2}}+\frac{k^{2}}{a^{2}} \cdot \varphi_{k}^{2}-\frac{16}{3} \cdot c_{1} \cdot B^{4}\right)
\end{aligned}
$$

The last line of Equation (12) states that, if we apply it to the Pre Planckian to Planckian regime, that there will be a change in the energy, which we will call

$$
\Delta E \approx\left(\frac{3}{8 \pi G} \cdot \frac{\kappa}{a^{2}}+\frac{k^{2}}{a^{2}} \cdot \varphi_{k}^{2}-\frac{16}{3} \cdot c_{1} \cdot B^{4}\right)
$$

We then will call this shift in energy, as equivalent to a change in KINETIC energy, and then reference the Virial theorem [19] which in a general form, will be interpreted as

$$
\langle\psi| \text { Kinetic-Energy }|\psi\rangle=\langle\psi| r \cdot \nabla V(\text { Potential-energy })|\psi\rangle
$$

Leading to

$$
\begin{aligned}
& \langle\psi|\left[\text { Kinetic-Energy } \approx\left(\frac{3}{8 \pi G} \cdot \frac{\kappa}{a^{2}}+\frac{k^{2}}{a^{2}} \cdot \varphi_{k}^{2}-\frac{16}{3} \cdot c_{1} \cdot B^{4}\right)\right]|\psi\rangle \\
& \approx\langle\psi|\left(r \cdot \nabla\left[V(\text { Potential-energy }) \approx c_{2} / r^{\alpha}\right]\right)|\psi\rangle
\end{aligned}
$$

In the Pre Planckian to Planckian space time, we will approximate, in the instant before time is initialized, formally, the mean value theorem as to the computed values of both the Left and right hand sides of Equation (14) and Equation (15) with the results that we obtain

$$
\begin{aligned}
& \left(\frac{3}{8 \pi G} \cdot \frac{\kappa}{a^{2}}+\frac{k^{2}}{a^{2}} \cdot \varphi_{k}^{2}-\frac{16}{3} \cdot c_{1} \cdot B^{4}\right) \approx-\alpha / r^{\alpha} \equiv-\alpha /(\text { Planck-length })^{\alpha} \\
& \Leftrightarrow\left[\alpha /(\text { Planck-length })^{\alpha}\right] \approx \frac{16}{3} \cdot c_{1} \cdot B^{4}-\left(\frac{3}{8 \pi G} \cdot \frac{\kappa}{a^{2}}+\frac{k^{2}}{a^{2}} \cdot \varphi_{k}^{2}\right)
\end{aligned}
$$

Here, the magnetic field would be determined in part by the value of $B$, as given in [20], and the scale factor $a$, is given by Equation (2) and Equation (4), and $\varphi_{k}$ is given by Equation (8).

This shows in part that $\alpha$ is no longer strictly real valued but is strongly influence by the input from $\varphi_{k}$, i.e. which has real and imaginary components.

We will next, in Part 3 of this document conclude with a specific statement as to how the 3-body problem, and in fact other experimental science could be impacted by Equation (25) and a careful reading of [3], page 134, has a very carefully done section on the so called Lagrangian equilateral triangle and possible orbits 3 body problem, and [21] has its section on the KAM theory, page 15, 
which are subsequently modified, and which may yield more rigorous simulations, in the computer numerical sense which in turn could give us more useful input into experiments.

\section{How to Reconcile Our Developments with Ascertaining Limitations and Also Improvements on the 3 Body Problem of Classical Physics (Plus Its Quantum Analogues)}

What we are doing is to consider both [3] and [21] in terms of KAM theory, [21], as of page 15, have in its classical mode a highly restricted set of equations, as given by having the following quote.

From [21],

Quote

Now, the KAM theorem tells us that when the system is slightly perturbed, most of the invariant tori are not destroyed but only slightly shifted in the phase space. This has important implications on stability of orbits in the general and restricted three body problem. The proof of the KAM theorem by Moser [1962] and Arnold [1963] also demonstrated that convergent power series solutions exist for the three-body (as well as for the n-body) problem. The KAM theorem seems to be very useful for studying the global stability in the three-body problem [Robutel, 1993a, Montgomery, 2001, Sim'o, 2002]; however, some of its applications are limited only to small masses of the third body.

The limitation as stated as of KAM theory is that MOST of the time, we have that the KAM results require a third body to be low mass.

This is a classical dynamical system result. What we should endeavour through judicious application of Equations (25) and (29) is to remove dependence upon the smallness of the third mass, and to examine if this can still, with a nonnon-trivial third mass recover still much of the stability analysis.

Later, at an appropriate time, this question in terms of a serious application of the value of Equation (16) will be pursued.

Secondly, as of [3], the section given on page 154, entitled "6.4 Orbital changes in encounters with planets", which is a restricted 3 body problem, frequently is used as to the interaction of saying comets (comparatively small mass) with a planet, circulating the Sun, where we have 2 "massive" masses, and the third body, in this case a comet, which gives usually parameters of how a hyperbolic orbit for a comet, i.e. one which enters in a planet-Sun system, impacted via how the parameters of saying a hyperbolic trajectory of a minor mass object (comet) impacted in a simple solar system model.

We hope that by judicious investigation of the arguments given as of to Equation (16), the restrictions as to the smallness of the "third" mass may be partly ameliorated.

If this is done, and it will require through investigations, then a template as to how to reliably simulate $\mathrm{N}$ bodies interacting, may be a doable problem, but first of all, to do this, it should be seen if the classical KAM problem may be general- 
ized beyond its present strictures.

And, now for an overall conclusion:

We conclude that a worthy application of our techniques is in investigating if restrictions as to the KAM results, and restricted small mass of a third body in terms of interaction with two other bodies are doable and made more likely via Equation (16). We argue that Equation (16) in its limiting characterization may allow certain classical 3 body simulations more flexibility than what exist presently. We also leave the door open as to other applications of Equation (16), especially in early universe conditions.

In closing, we also refer to two further references which may allow for additional research refinement of the NLED paradigm which may allow for good applications of our idea, i.e. [21] [22] [23] which may further refine our results and which bear consideration.

This should be also an extension of what we have been doing in [24] and we will be attempting to find such a connection in the regime of space-time in the Pre Planckian to Planckian regime transition where we use NLED approximations as to scale factor, assuming in this regime a relatively constant, presumably extraordinarily hot initial cosmological temperature.

The details of all this, as well as other details pertinent to the creation of presumed mini black holes will be the subject of our inquiry as to modified gravity. See [25] for recent work by Dr. Corda and others which may be accessed immediately for useful ideas on the creation of initial black holes in the near future which we will try to reconcile with our modified gravity ideas. We will try to reconcile [24] and [25] as part of our inquiry as to modifying Equation (16) in the near future.

\section{Acknowledgements}

This work is supported in part by National Nature Science Foundation of China grant No. 11375279.

\section{Conflicts of Interest}

The author declares no conflicts of interest regarding the publication of this paper.

\section{References}

[1] Mukhanov, V. (2005) Physical Foundations of Cosmology. Cambridge University Press, New York, New York, USA. https://doi.org/10.1017/CBO9780511790553

[2] Beckwith, A. (2015) Nonlinear Electrodynamics and Modification of Initial Singularities, and Dark Matter and Dark Energy Affecting Structure Formation in the Early and Later Universe. http://iopscience.iop.org/article/10.1088/1742-6596/626/1/012058/pdf

[3] Valtonen, M. and Karttunen, H. (2016) The Three-Body Problem. Cambridge University, Cambridge.

[4] Guth, A. (2007) Eternal Inflation and Its Implications. Journal of Physics A, 40, 6811-6826. http://arxiv.org/abs/hep-th/0702178 
https://doi.org/10.1088/1751-8113/40/25/S25

[5] Camara, C.S., de Garcia Maia, M.R., Carvalho, J.C. and Lima, J.A.S. (2004) Nonsingular FRW Cosmology and Non Linear Dynamics.

http://arxiv.org/pdf/astro-ph/0402311.pdf

[6] Weinberg, S. (1972) Gravitation and Cosmology, Principles and Applications of the General Theory of Relativity. John Wiley and Sons, New York.

[7] Corda, C. and Cuesta, H. (2010) Removing Black Hole Singularities with Non Linear Electrodynamics. Modern Physics A, 25, 2423-2429.

[8] Penrose, R. (1975) Gravitational Collapse and Space-Time Singularities. Phys. Rev. Lett., 14, 57.

[9] Penrose, R. (1969) Gravitational Collapse: The Role of General Relativity. NC1, 252-276.

[10] Park, D.K., Kim, H. and Tamarayan, S. (2002) Nonvanishing Cosmological Constant of Flat Universe in Brane world Scenarios. Physics Letters B, 535, 5-10. https://doi.org/10.1016/S0370-2693(02)01729-X

[11] Kobayashi, T. and Seto, O. (2014) Polynomial Inflation Models after BICEP2. Physical Review D, 89, 103524. http://arxiv.org/abs/1403.5055 https://doi.org/10.1103/PhysRevD.89.103524

[12] Adamek, J., Clarkson, C., Durrer, R. and Kunz, M. (2015) Does Small Scale Structure Significantly Affect Cosmological Dynamics? Physical Review Letters, 114, 051302. http://arxiv.org/abs/1408.2741 https://doi.org/10.1103/PhysRevLett.114.051302

[13] Guth, A. (1982) Phase Transitions in the Early Universe. 171-204, In: Gibbons, G., Hawkin, S. and Siklos, S., Eds., The Very Early Universe, Proceedings of the Nuffield Workship, Cambridge, 21 June-9 July 1982, Cambridge University Press, 1982, Cambridge, UK.

[14] Lloyd, S. (2005) A Theory of Quantum Gravity Based on Quantum Computation. http://arxiv.org/abs/quant-ph/0501135

[15] Stahl, C. On Early and Late Phases of Acceleration of the Expansion of the Universe. $\mathrm{PhD}$ Thesis, International Relativistic Astrophysics. https://arxiv.org/pdf/1702.05630.pdf

[16] Beckwith, A. (2017) How a Minimum Time Step and Formation of Initial Causal Structure in Space-Time May Void the Penrose Singularity Theorem, as in Hawking's and Ellis's 1973 Write Ups. http://vixra.org/abs/1705.0213

[17] Padmanabhan, T. (2006) Volume 8: An Invitation to Astrophysics. World Scientific Series in Astronomy and Astrophysics, World Scientific, Singapore.

[18] Carroll, S. (2016) Spacetime and Geometry. Pearson India, New Deli, Republic of India.

[19] Gasiorowitz, S. (1974) Quantum Physics. $1^{\text {st }}$ Edition, John Wiley and Sons, New York, New York.

[20] Beckwith, A. (2017) How to Determine a Jump in Energy Prior to a Causal Barrier, with an Attendant Current, for an Effective Initial Magnetic Field. The Pre Planckian to Planckian Space-Time. http://vixra.org/abs/1707.0250

[21] Musielak, Z.E. and Quarles, B. (2014) The Three Body Problem. Reports on Progress in Physics, 77, Article ID 065901. https://arxiv.org/abs/1508.02312 https://doi.org/10.1088/0034-4885/77/6/065901

[22] De Lorenci, V.A., Klippert, R., Novello, M. and Salim, J.M. (2002) Nonlinear Elec- 
trodynamics and FRW Cosmology. Physical Review D, 65, 063501.

https://doi.org/10.1103/PhysRevD.65.063501

[23] Corda, C. and Mosquera Cuesta, H.J. (2011) Inflation from $R^{2}$ Gravity: A New Approach Using Nonlinear Electrodynamics. Astroparticle Physics, 34, 587-590. https://doi.org/10.1016/j.astropartphys.2010.12.002

[24] Beckwith, A. (2018) How a Laser Physics Induced Kerr-Newman Black Hole Can Release Gravitational Waves without Igniting the Black Hole Bomb (Explosion of a Mini Black Hole in a Laboratory).

https://www.researchgate.net/publication/327867961_HOW_A_Laser_Physics_Ind uced_KERR-NEWMAN_BLACK_HOLE_CAN_RELEASE_GRAVITATIONAL_W AVES_without_igniting_the_Black_Hole_bomb_explosion_of_a_mini_black_hole_ in_a_laboratory

[25] Corda, C. and Feleppa, F. (2018) Black Hole as Gravitational Hydrogen Atom by Rosen's Quantization Approach.

https://www.researchgate.net/publication/328224956_Black_hole_as_gravitational_ hydrogen_atom_by_Rosen's_quantization_approach 